\title{
Riesgo, ambiente y percepciones en una comunidad rural totonaca
}

\section{Risk, environment and perceptions in a Totonacan rural community}

\author{
María del Carmen Vergara-Tenorio \\ Juan Roberto Cervantes-VÁzQueZ**
}

\begin{abstract}
In this study we analyse the environmental risk and its social perception in a rural community of the Sierra Totonaca in Mexico. This population has suffered a great ecological impact due to the change in land usage and deforestation. The population has developed two strategies to diminish their risk: broaden their livestock and farming productive base and migration to the city. We analyse the consequences of these actions and their relationships in a sociological and ethnographical context.
\end{abstract}

Keywords: rural environment, Totonacan community, risk perception, environmental risk.

\section{Resumen}

En esta investigación se analiza el riesgo ambiental y su percepción social en una comunidad rural de la sierra totonaca en México. Esta población ha sufrido grandes impactos ecológicos debido al cambio de uso del suelo y la deforestación. Los pobladores han desarrollado dos estrategias para aminorar el riesgo: ampliar su base agropecuariaproductiva y migrar hacia las ciudades. Se analizan las consecuencias de estas acciones y las relaciones dentro de un contexto sociológico y etnográfico.

Palabras clave: ambiente-rural, comunidad totonaca, percepción de riesgo, riesgo medioambiental.

* Universidad Veracruzana, México. Correos-e: cvergara@uv.mx, roberto466@yahoo.com.mx. 


\section{Introducción}

El riesgo es algo con lo que comúnmente estamos en contacto en nuestra vida cotidiana; éste se presenta con el solo hecho de darnos un baño o viajar en automóvil. De ahí que existen diferentes tipos de riesgo a los que nos enfrentamos día a día. En la actualidad uno de los riesgos más importantes es el ambiental, el cual existe cuando nuestro entorno o el de cualquier ser vivo se modifica de manera que pone en peligro la vida y por lo tanto trastoca la cotidianidad (Toledo, 2003: 23). Este tipo de riesgo nos afecta no sólo de manera individual, sino de forma colectiva y lo vemos manifestado en fenómenos como la construcción y operación de plantas nucleoeléctricas o hidroeléctricas, el aumento progresivo de la utilización del automóvil, las alteraciones provocadas por cambios abruptos en el uso del suelo y la pérdida de especies, la utilización de productos químicos para acelerar la producción agraria, el aumento en industrias contaminantes y las enfermedades de las comunidades humanas y hábitats naturales (Beck, 1999: 212; Leff, 2002: 47).

Desde un punto de vista etnográfico, en esta investigación se aborda principalmente el tema del riesgo relacionado con el ambiente y la percepción social que tiene una comunidad rural de la sierra totonaca veracruzana sobre su entorno. En la búsqueda de mejores condiciones económicas, esta población como otras de la región ha sufrido grandes impactos debido al cambio de uso del suelo y la deforestación. Como ejemplo de esto se tiene la adaptación de grandes extensiones de terreno para uso ganadero, lo cual contribuye a que el estado tenga aproximadamente $81 \%$ de su suelo dedicado a la ganadería, con un total de 5'936,840 ha (INEGI, 1998). Además, los grupos étnicos de la zona basan su economía en la agricultura que se realiza en áreas poco propicias para este fin, demandando cada vez mayor cantidad de suelo productivo. Esta situación ha resultado en la existencia de un riesgo constante en el lugar y el deterioro ambiental de la zona. En este trabajo se explica cómo definen las personas el concepto de riesgo relacionado con el uso de los recursos naturales y se asume que el riesgo ambiental es aquella situación donde el entorno de una población humana pierde la capacidad de sostener su modo de vida (Bell y Morse, 1999: 60; Vaugh, 2001: 79). Sin embargo, es importante reconocer que el riesgo ambiental es una idea socialmente construida (Douglas y Wildavsky, 1982: 22). Las diferentes sociedades maximizan o minimizan distintos tipos de ries- 
gos, lo que se traduce en prácticas específicas para asegurar y mantener una buena calidad de vida (Vergara, 2000). Desde está perspectiva resulta interesante estudiar el tema del riesgo ambiental, ya que si partimos de la idea de preservar y no sobreexplotar los recursos naturales para el desarrollo sustentable, es fundamental entender las consecuencias de los modelos de crecimiento implementados aun en las pequeñas comunidades rurales. Por tanto, la investigación en un lugar específico contribuye a reflexionar acerca de las concepciones locales, sobre el crecimiento económico, la modernización y las situaciones de riesgo ambiental a corto, mediano o largo plazos.

Una de las cuestiones más importantes para los fines de la investigación fue averiguar exactamente qué concepto tienen los miembros del Poblado 1 sobre el medio ambiente o la naturaleza, y qué tan familiarizados están con estos términos. Además se investigó la construcción de la imagen que esta comunidad tiene de la naturaleza, considerando siempre su vocabulario y terminología desde un punto de vista etnográfico. Asumimos que a partir de estas concepciones los individuos creamos relaciones particulares, conocimiento y modificaciones con aquello que nos rodea, es decir, se realiza la construcción social de los conceptos (O’Connor, 2001: 123). En la investigación nos interesaba saber la idea de riesgo que tienen los habitantes, tomando en cuenta el uso de los recursos y del medio ambiente. Consideramos que los miembros del Poblado 1 se encuentran inmersos en la sociedad de riesgo, la cual abarca los temas ambiental, social, laboral y económico (Toledo, 2003). Es decir, las poblaciones locales también comparten problemas globales y es necesario contextualizar sus procesos de desarrollo y acciones para evitar el riesgo. Los individuos se encuentran sujetos tanto a presiones, patrones y políticas locales como regionales o internacionales. Por tanto, asumimos que el riesgo ambiental es la situación donde el entorno ecológico de una población humana pierde la capacidad de sostener su modo de vida, ya sea por causas antropogénicas o naturales (Bell y Morse, 1999: 46; Vaugh, 2001: 61). Para fines de la investigación entendemos que la insostenibilidad recae en aspectos como el alimenticio, el empobrecimiento del suelo, el recrudecimiento o aparición de algunos fenómenos naturales como los cambios bruscos del clima que se manifiestan en prolongadas sequías o abundantes lluvias para los cuales la población ya no tiene capacidad de respuesta, alterando de manera 
significativa su ciclo productivo; o también se refleja en las cuestiones de salud y enfermedad.

Para la investigación fue importante reconocer que la población es de tipo rural y que una de sus principales actividades económicas es la agricultura, la cual se ve afectada directamente por la cuestión ambiental, que a su vez influye en la dinámica individual y colectiva de la vida cotidiana de sus habitantes. Otro aspecto esencial que consideramos fue el tipo de acceso a los recursos naturales como tierra, agua, bosque e incluso al conocimiento de usos de plantas medicinales o alimenticias; ya que esto señala si un grupo está en mayor riesgo que otros en términos alimenticios. El trabajo también se enfocó en mostrar las diferentes visiones que se tienen en la misma comunidad sobre el problema ambiental, las formas en que se negocian los recursos y las estrategias de adaptación a la situación de riesgo, destacando las expectativas que tienen los pobladores sobre su futuro personal, familiar o comunitario. Finalmente, se contrasta la situación ambiental, social y económica para buscar opciones de recuperación, mejoramiento o mantenimiento del lugar de acuerdo con el pensamiento predominante.

\section{Descripción de la zona de estudio}

La investigación se llevó a cabo en una población del Totonacapan de la región de Papantla, que denominaremos Poblado $1 .{ }^{1} \mathrm{Se}$ eligió esta comunidad porque es representativa de la región, de los problemas y peculiaridades de los habitantes de la zona y por la preocupación de riesgo ambiental expresada por la población. El Totonacapan se extiende desde la sierra y tierras bajas del norte de Puebla hasta el centro y parte del norte de Veracruz, y comúnmente se concibe como una de las áreas más ricas del país, con una vegetación predominante de bosque tropical perennifolio, donde abundaban especies maderables y animales. Sin embargo, esta zona también es un lugar donde los recursos naturales se han manejado intensivamente debido a los ajustes de la producción económica regional, con la consecuencia de una inminente amenaza a la biodiversidad local y al suelo útil (Dávila, 2000: 89; Ramírez, 2002: 62). La sierra de Papantla-conformada por los municipios de Zozocolco de Hidalgo, Coxquihui, Coyutla y Chumatlán- se caracteriza por asentamientos huma-

${ }^{1}$ El nombre de la comunidad se mantendrá en el anonimato por respeto a las personas entrevistadas y porque existen proyectos de investigación en marcha en la zona. 
nos predominantemente concentrados-dispersos y la comunicación entre los diversos pueblos es mediante caminos de terracería y veredas (Ichón, 1973: 400; Ortiz, 1990: 23; Gomezjara, 1998: 78). El transporte es colectivo y comunica a las cabeceras municipales y a las ciudades de Papantla, Poza Rica y Tuxpan (Pemex; 1999: 13); para las poblaciones más alejadas se utilizan camionetas de peaje (Segura; 2002: 25). El municipio de Zozocolco, donde se encuentra el Poblado 1, está catalogado como de alta marginalidad y cuenta con un paisaje principalmente agroforestal, compuesto de plantaciones pequeñas de cafetos y de cultivos anuales de frijol, chile y maíz. Además tiene grandes extensiones dedicadas a la ganadería cuya producción es en su mayoría para autoconsumo y sólo una pequeña parte para comercialización (Pemex, 1999: 67). La cabecera municipal se fundó oficialmente el 2 de agosto de 1966, tras un proceso que data de 1815 (Gomezjara, 1998: 57). Por otra parte, la población en cuestión es de fundación más reciente. Inicialmente, el litigio por los terrenos comenzó en 1932, en 1963 se constituyó como ejido y en 1976 se realizó el deslinde definitivo de las tierras (Gomezjara; 1998: 34). A cada uno de los 41 ejidatarios se les concedió aproximadamente 3.5 ha de parcela para sembrar y un solar de unos $20 \mathrm{~m} \times 20 \mathrm{~m}$ para su casa.

Actualmente la comunidad de Poblado 1 se constituye por 350 habitantes, los cuales están organizados en ejidatarios y avecindados. Estos últimos sólo cuentan con el terreno de su casa y un pequeño solar y no poseen parcela a menos que la compren. La fiesta patronal del pueblo es la celebración más importante y se realiza por medio de mayordomías. La comunidad cuenta con una iglesia construida por los pobladores y tiene tres niveles educativos: primaria, telesecundaria y telebachillerato. Las instalaciones para los primeros dos niveles se construyeron por medio del trabajo colectivo. Las clases de bachillerato para todos los semestres del ciclo escolar se imparten en una bodega. El servicio médico se encuentra en otra población cercana, pero los habitantes prefieren ir a la cabecera municipal. La organización política del poblado incluye a las autoridades ejidales y a las civiles. Las autoridades ejidales sólo las pueden ocupar ejidatarios y comprenden: presidente del comisariado ejidal, secretario, tesorero, secretarios, subagente y secretario. El cargo de presidente del comisariado ejidal y el de tesorero, con sus respectivos secretarios, únicamente lo ocupan ejidatarios y son quienes junto con los otros ejidatarios deciden las cooperaciones monetarias o 
trabajo colectivo, faenas, que se deben realizar y la tenencia de la tierra. Las autoridades civiles (subagente municipal y secretario) pueden estar a cargo tanto de avecindados como de ejidatarios. También existen las sociedades de padres que se organizan en los tres niveles escolares y cualquiera puede formar parte de ellas siempre y cuando tengan hijos inscritos en la escuela.

\section{Metodología}

La investigación sobre el tema de riesgo ambiental en el Poblado 1 se realizó en varias etapas desde junio de 2003 hasta septiembre de 2004. La metodología que se utilizó fue de tipo cualitativo-etnográfico. En primer lugar se hizo un recorrido por varias comunidades del municipio de Zozocolco y se hicieron visitas exploratorias para seleccionar una comunidad. Posteriormente se decidió el Poblado 1 como lugar de estudio porque, de acuerdo con los habitantes del lugar, es una comunidad donde se vive una situación de riesgo y es representativa de las comunidades totonacas del área. Es decir, la comunidad es de tipo rural, sus pobladores pertenecen a la etnia totonaca y sus condiciones son marginales. En esta población se aplicó una encuesta exploratoria de tipo aleatorio a 20 personas ( 11 hombres y 9 mujeres) con el fin de tener un contacto más personal con los habitantes y para habituarlos a la presencia de los investigadores. La información recabada en la encuesta representa datos con un nivel de confianza de $95 \%$ y un intervalo de confianza de 20 puntos. La información se digitalizó en el programa estadístico SPSS para su análisis y graficación.

En la segunda etapa se hicieron cuatro visitas de campo cortas con el objetivo de conocer a los pobladores de la comunidad y adaptar la investigación de acuerdo con el contexto de la población. También uno de los investigadores realizó una estancia de un mes con el propósito de tener un contacto cotidiano con las personas del pueblo. Una actividad importante en este periodo fue localizar informantes clave que proporcionaran datos sobre la historia del pueblo, la estructura política de la comunidad, información sobre grupos sociales, su conformación y actividades. Como tercera etapa se realizaron 26 entrevistas semiestructuradas (17 hombres y 9 mujeres) que duraron un promedio de dos horas cada una y abarcaron diferentes puntos de vista sobre el tema del riesgo ambiental, así como las perspectivas presentes y futuras de la población. Las entrevistas se realizaron por me- 
dio de intérpretes, ya que aunque la mayoría de las personas es bilingüe, se expresan mejor en su lengua. Asimismo, las entrevistas se transcribieron en un procesador de textos -según las notas, observaciones y/o grabaciones- para su análisis y consulta. Es esencial mencionar que la perspectiva histórica del manejo de los recursos naturales en México, y en el estado de Veracruz, ha ocasionado políticas públicas que indudablemente han contribuido a un manejo no adecuado. Sin embargo, la investigación se enfoca a analizar las cuestiones y las perspectivas que los propios miembros de la comunidad tienen sobre sus realidades locales.

\section{Resultados}

\subsection{Estructura de la comunidad}

La mayoría de los habitantes (90\%) del Poblado 1 es bilingüe, hablan totonaco y español. Un 10\% sólo habla totonaco y en su mayoría son las personas de más edad (50 años en adelante) que conservan la forma tradicional de vestimenta. Para los varones ésta consta de pantalones, camisa de manta y huaraches; para las mujeres de vestido blanco, bordado con listón y sandalias o descalzas (Ichón, 1973: 401). Como se pudo constatar en las observaciones de campo, la mayoría de la población de 15 a 39 años ya no conserva este modo de vestir, el cual sustituyen, los hombres, por camisas de algodón, pantalón de mezclilla y botas o huaraches; las mujeres, por vestidos de colores brillantes, ocasionalmente bordados, y sandalias. También cabe mencionar que todos los habitantes se consideran totonacos, aun en casos en que confesaron no hablar muy bien la lengua. En cuanto a preparación y escolaridad, en la comunidad el grueso de la población adulta sólo concluyó algún grado de educación primaria, pocos son los que carecen de algún tipo de escolaridad (5\%). De acuerdo con la información recabada, $70 \%$ de los encuestados asistieron a la primaria, $15 \%$ a la secundaria y $10 \%$ a la preparatoria. Sin embargo, pese a que cuentan con el telebachillerato, pocos son los que egresan (21\%, respecto de los que ingresaron, $79 \%$ en 2003) debido a que prefieren ir a trabajar a México o Puebla que continuar estudiando. Por tal situación, en época de vacaciones se observa que en el pueblo no hay jóvenes de entre 18 a 26 años. 


\subsection{Actividades laborales}

De acuerdo con las encuestas y las entrevistas, 35\% de los pobladores son campesinos, $15 \%$ son campesinos y tienen otra ocupación, 40\% se dedican al hogar y 10\% son estudiantes. Las actividades laborales primordiales para los hombres son las agrícolas, aunque algunos cuentan con un trabajo secundario. Los varones trabajan su parcela, rentan terrenos o se emplean como jornaleros. Se observan sobre todo dos tipos de uso de suelo: la cría de ganado y el cultivo de maíz. La cría de ganado se realiza en grandes extensiones que pertenecen a personas que viven en Papantla o Poza Rica. Principalmente se siembra maíz pero existen otros cultivos como pimienta y café. Este último tuvo gran auge pero se abandonó por los bajos precios en el mercado y las plagas. Por otra parte, las mujeres se dedican sobre todo a las actividades del hogar y a ayudar a sus maridos durante la cosecha y la limpia del maíz. Los jóvenes en su mayoría asisten a las escuelas de la comunidad, algunos incluso estudian en las cabeceras municipales; la mayor parte de quienes salen a otras escuelas son mujeres. En contraparte, los jóvenes varones combinan sus actividades escolares con las labores del campo y trabajan en las parcelas o emigran. Para ellos la ayuda en la parcela familiar es casi obligatoria, al llegar a los 12 o 15 años tienen el deber de aprender a trabajar en el campo.

Los avecindados se emplean como jornaleros o cultivan algún terreno rentado para obtener un ingreso y maíz. Además, entre los miembros de la comunidad se llega a establecer un pequeño mercado interno para la adquisición de grano. El uso extensivo de la tierra ha ocasionado el desmonte continuo de la zona, causando la desaparición paulatina de especies vegetales y animales nativas como el armadillo, el tigrillo o gato montés y el venado. Por lo que, de acuerdo con los pobladores, se ha desarrollado un proceso de empobrecimiento del suelo debido al uso de químicos y fertilizantes lo cual disminuye la cantidad y calidad de los productos cultivados. El aprovechamiento de recursos maderables se hace principalmente de manera interna. Las personas del pueblo utilizan la madera como material de construcción para casas, muebles o como leña, por lo que la extracción externa es mínima. Respecto a la conservación y reforestación mostraron cierto interés, ya que para ellos significaría una manera más de conseguir un ingreso. Por ejemplo, los pobladores utilizarían los árboles como madera para venta, que según su opinión, se cotiza 
muy bien en las ciudades. Asimismo, tienen cierto rechazo a una reforestación o conservación que no les ayude a mejorar sus condiciones económicas de vida.

\subsection{Conceptualización de la naturaleza}

En nuestra investigación registramos que 90\% de las personas han observado una pérdida de plantas y $95 \%$ mencionaron pérdida de animales. La mayoría de los informantes concuerdan con que se ha dado una disminución considerable de la naturaleza nativa en comparación con el paisaje original. Incluso los jóvenes evocan narraciones de sus padres, abuelos o personas ancianas del pueblo que expresan que ya no se encuentran plantas y animales que existían antes. En algunos casos mencionaron aspectos positivos de la pérdida de especies, ya que ha disminuido el peligro de ataque de algún animal ponzoñoso dentro de sus hogares. Pero en general todos los entrevistados destacaron que la pérdida de especies ha sido perjudicial para el poblado y principalmente para la agricultura, ya que la tierra poco a poco ha reducido su fertilidad, el grado de erosión aumenta y las plagas se hacen cada vez más resistentes a los plaguicidas.

Durante las entrevistas los campesinos expresaron claramente la necesidad de ayuda externa para solucionar problemas de producción. Además, fue evidente que los fenómenos causados por las alteraciones globales climáticas afectan directamente cómo las personas entienden su entorno. Por ejemplo, varios campesinos comentaron que hoy en día es más difícil determinar las épocas de siembra y cosecha debido a que las temporadas de lluvia y sequía varían constantemente, por lo que les resulta difícil establecer patrones. La mayoría de los entrevistados expresan estas alteraciones del ambiente local como cambios en el clima (80\%) o como aumento en la sequía (85\%). En el Poblado 1 los cambios se consideran negativos. De acuerdo con los entrevistados, ahora la época de sequía dura mucho más tiempo y no se puede anticipar. Asimismo observamos que las personas del pueblo muestran preocupación por la situación ambiental, pues opinan que afecta de manera dramática su vida cotidiana, su dinámica social y familiar. Cuando indagamos sobre la forma en que evalúan los problemas en México, el medio ambiente apareció como una preocupación relevante para los pobladores. Sus opiniones sobre los problemas del país se distribuyen de la siguiente manera: desempleo $20 \%$, seguridad $20 \%$, medio ambiente $15 \%$, eco- 
nomía 10\% y 35\% no tuvo opinión al respecto. En consecuencia, la investigación reveló que, en primer lugar, en el Poblado 1 hay una pérdida de hábitat y una degradación severa del suelo debido principalmente a los cambios drásticos en el uso del suelo, la intensificación de los monocultivos, el incremento de sustancias químicas, la deforestación y la pérdida de especies. Como reflejo de lo anterior podemos citar a un campesino de 40 años quien dijo: "Se producía más antes, ahora hay desgaste, para que se dé la cosecha se necesitan fertilizantes y perjudican la tierra".

\subsection{Estrategias de vida}

En comunidades rurales indígenas el riesgo provocado por las prácticas expansivas de explotación de la tierra no sólo pone en peligro la capacidad productiva, también la sustentabilidad de la vida (Beck, 1991: 16; Beck, 1999: 212; Roma, 2002: 23). Esta situación se detecta en el Totonacapan veracruzano, específicamente en el Poblado 1. Las situaciones de riesgo ambiental han forzado a los pobladores a crear estrategias para enfrentar los nuevos retos. Durante la investigación fue claro que las estrategias que los miembros del Poblado 1 utilizan varían: desde introducir nuevos cultivos a una tierra infértil hasta abandonar el pueblo. Con la primera estrategia las personas intentan reincorporar el cultivo tradicional de vainilla, pero para tener éxito necesitan recuperar el suelo y la vegetación. Otro cultivo que proponen es la caña de azúcar, lo que les daría resultados a corto plazo pero deterioraría aún más el suelo, ya que la caña es altamente destructiva y promovería la ideología de producir sin considerar las condiciones del lugar (INEGI, 1998; Leff, 2002: 56). La cría de ganado es la tercera opción productiva propuesta por los pobladores, pues la mayoría piensa que una solución a sus problemas es introducir pequeñas cantidades de ganado. Sin embargo, tener animales requiere de recursos monetarios, que la mayoría no posee. El ingreso semanal promedio de la comunidad va de 100 a 400 pesos, es decir, de 2 a 7 dólares al día por persona para vivir. Como segunda estrategia contra los riesgos, que están empezando a desarrollar los pobladores, es migrar sobre todo a las ciudades de México y Puebla. En general detectamos que $20 \%$ de los pobladores ha salido a trabajar a dichas ciudades y que $90 \%$ de ellos tienen al menos un familiar trabajando fuera del Poblado 1. Generalmente son los hijos varones quienes salen a trabajar ya sea de manera temporal, en periodos vacacionales o de forma definitiva. La principal fuente de empleo 
que obtienen es en los servicios de lavado de autos o estaciones camioneras urbanas. Según un estudiante de 25 años, "Los muchachos aprenden a trabajar la tierra por obligación, pero prefieren irse a Puebla y México a las fábricas y de lavacarros". Finalmente, cabe destacar que aquellas personas que manifestaron una preferencia por quedarse en el Poblado 1, lo hacen por el aprecio que tienen a su comunidad, a la tranquilidad y la poca violencia que se vive en el lugar.

\section{Discusión}

\subsection{Naturaleza, actividades productivas y sociedad de riesgo}

La continua interacción entre los miembros de una sociedad con el entorno natural crea una conceptualización particular sobre la naturaleza, al grado que se llega a convertir en un símbolo para dicho colectivo social y determina las relaciones con el ambiente (Beck-Gernsheim,1996: 139; Mires, 1996: 34). De esta manera, la concepción de domesticación, civilización y racionalización de la naturaleza primigenia nos ha llevado a perder nuestro sentido de escasez y a considerar a los recursos naturales como una fuente inagotable e incondicional (Vaugh, 2001: 78). En muchas zonas del Totonacapan se puede ver claramente la aplicación de esta concepción sobre la naturaleza. En el Poblado 1 existen varios factores que han provocado que la idea sobre la naturaleza, representada por lo que la gente conoce como monte, cambie completamente, al grado que las personas son capaces de expresar "que en su pueblo no hay naturaleza". Los factores que encontramos como aceleradores de este proceso son: la pérdida de la vegetación nativa para cultivo o ganadería, la sustitución de cultivos, el uso de tecnología inapropiada y el abuso de pesticidas.

Particularmente la existencia de grandes extensiones de terreno dedicadas a la ganadería o a la agricultura extensiva en el Totonacapan veracruzano, nos da pauta para reflexionar sobre la forma de producción en el campo mexicano. Claramente podemos definir una disyuntiva entre la forma tradicional de producir y la necesidad de adaptarse a las exigencias del mercado. Aunadas a estas transformaciones se encuentra una más: el abandono del sistema de tumba y quema por el uso de herbicidas. El cambio lo motivó el incremento en la demanda de maíz para abastecer las necesidades propias o las del mercado y está apoyado por diversos programas gubernamentales con el fin de aumentar 
y modernizar la producción agrícola (Pemex, 1999: 38; Ramírez, 2002: 87).

Sin embargo, el control de plagas es cada vez más difícil por la resistencia natural que éstas crean y que a su vez disminuye la disponibilidad de tierras sanas, provocando una dependencia hacia soluciones más tecnificadas y externas con el consecuente desplazamiento de las técnicas de manejo local (Dubos, 1985: 13; Leff, 2002: 75; Foucault, 2004: 42). Esto se refleja en lo que manifestó un campesino de 48 años de edad: "Las plagas se dan cada cosecha y es necesario utilizar químicos, con los años son más difíciles y aparecen nuevas plagas". En el estudio es evidente que los pequeños productores están tratando de producir bajo las reglas del mercado comercial, sin cuestionar las posibilidades reales de estas actividades en su localidad o las consecuencias ambientales a futuro. De esta forma se sigue ampliando la extensión de tierra cultivable y se utiliza una gran cantidad de agroquímicos, lo que en definitiva ejerce una mayor presión al ambiente.

Aunque la siembra de maíz es la actividad más importante en el Poblado 1, vale la pena señalar que el café y la vainilla fueron dos cultivos muy importantes para la economía del lugar. El café llegó a ser casi exclusivamente la principal fuente de ingresos para los ejidatarios (Pemex, 1999: 54; Ramírez, 2002: 67). Durante las entrevistas fue claro que a partir de 1996 y 1997 se comienza a abandonar este cultivo debido a los bajos precios, a la clasificación como café de baja altura y a la invasión de la plaga de broca entre 1998 y 2000. La problemática causada por la devaluación del café fue tan grave, que el pueblo entero llegó a vivir una profunda crisis económica, la cual motivó que muchos hombres migraran para equilibrar y aumentar su nivel de vida. Por otro lado, el cultivo de vainilla ha sido importante para el pueblo y para la zona del Totonacapan, ya que tiene una gran demanda nacional e internacional. Pero este cultivo se ha abandonado en diversos periodos, principalmente por la entrada de sustitutos de bajos precios provenientes de Asia, provocando la caída del precio del producto. También se ve afectado por robos, variaciones del clima y la pérdida de tierra adecuada para el cultivo por la expansión de la ganadería (Gomezjara, 1998: 37; Ramírez, 2002: 89).

En este estudio también se revela el concepto de naturaleza productiva que implica que se aceptan acciones para mejorar o conservar el ambiente, siempre y cuando se obtenga un beneficio directo/económico de la tierra. Debido a la situación tan difícil que viven los habitantes de la zona y al hecho de que tienen 
que elegir entre conservar y sobrevivir bajo condiciones de marginación, en el Poblado 1 observamos una constante necesidad de capitalizar la naturaleza. La reducción de especies sugiere la pérdida del hábitat y la disminución en la calidad de vida productiva y económica de los habitantes del poblado. Por ejemplo, un ama de casa de 30 años explica que "Las plantas de monte son cada vez más difíciles de conseguir". Otra mujer de 60 años reitera que "Sí han desaparecido animales salvajes y el monte para leñar y sembrar". En otros términos, este riesgo ambiental agudiza el nivel de pobreza porque se trata de una comunidad rural que depende de la agricultura, además de que también propicia un riesgo de tipo laboral (Toledo, 2003: 34), ya que se pierde la fuente principal de producción y sustento.

\subsection{El riesgo y el desarrollo}

El problema del riesgo ambiental no se puede entender sin considerar el tema del desarrollo sustentable, el cual busca disminuir los riesgos y pretende incrementar los niveles de vida de las sociedades humanas, considerando el uso adecuado de los recursos. Sin embargo, el desarrollo sustentable es muy difícil de conseguir debido a las implicaciones sociopolíticas, el necesario cambio de valores y la concepción de acciones en el mediano y largo plazos. De esta manera, las personas se encuentran en la disyuntiva de proteger su medio ambiente o sobrevivir, lo cual da la pauta para soportar los procesos económicos de prácticas capitalistas, sin considerar al medio ambiente (Leff, 2002: 45; Toledo, 2003: 29). En este sentido, y de acuerdo con las entrevistas que hicimos a autoridades del municipio de Zozocolco de Hidalgo, es claro que el concepto de desarrollo sustentable se ha incorporado al discurso oficial, las autoridades definen al desarrollo como: "la no explotación, sino el uso del recurso natural y obtener beneficios sin comprometer el futuro. Además de una protección del suelo, mantos acuíferos, áreas arboladas y especies de flora y fauna. Bajo este concepto se pretende conservar lo que se tiene a nivel municipal y restaurar lo que se ha perdido" (Entrevista núm. 7, autoridades municipales).

Sin duda éste es un cambio positivo, pero que no se refleja en un cambio en la mentalidad de la mayoría de los pobladores. Nuestra estancia en el Poblado 1 confirma que la gente que depende de la producción agrícola no ha cambiado de manera sustancial su forma de pensar y actuar en lo que concierne a la naturaleza, aunque discursivamente muestren un mayor interés por 
este aspecto. Esto se debe a que la producción agraria sigue guiada por el mercado sin evaluar las posibilidades reales de producción ni valorar a las especies nativas. En el municipio de Zozocolco se han hecho algunos esfuerzos por revertir estas tendencias, pero ninguna ha tenido impacto en el Poblado 1, ya sea porque la comunidad no se encuentra organizada o no se ha unido a los programas. De hecho, pudimos comprobar que $10 \%$ de los pobladores cuenta con cultivos alternativos, $5 \%$ han tenido contacto con algún vivero, $30 \%$ han realizado actividades de reforestación y $55 \%$ no participa en alguna actividad para mejorar el ambiente. Sin embargo, cabe señalar que cuando les preguntamos simplemente si participaban en acciones para preservar el medio ambiente, $75 \%$ declaró que no participaba, en contraste con $25 \%$ que sí lo hace.

Estos datos contrastan con la fuerte preocupación expresada anteriormente, donde consideran al medio ambiente como el tercer problema de México. Sin embargo, la poca participación se justifica porque prefieren buscar soluciones a corto plazo que les permita sostener a su familia o por ignorancia respecto a acciones para preservar el medio. La mayoría de los habitantes del Poblado 1 confiesan que generalmente no hay una comunicación entre los pobladores sobre lo que se piensa hacer para revertir el estado de riesgo en el que se encuentran. Asimismo, a través de las entrevistas, los pobladores y el gobierno municipal expresaron que la entrada de maquiladoras y fábricas de algún tipo ayudarían sustancialmente a la economía del lugar. También dijeron que la migración de los jóvenes se vería frenada y que se crearía un mayor arraigo a la comunidad por parte de las futuras generaciones. Aunque también manifestaron su falta de conocimiento sobre el impacto ecológico, consideran que con la tecnología adecuada el deterioro ambiental sería mínimo. Es claro que estas concepciones pertenecen al viejo discurso industrial del desarrollo que se promovió durante tanto tiempo en nuestro país (Villarreal, 2000: 15). Por último podemos decir que en esencia el discurso del desarrollo sustentable no es distinto del viejo discurso de desarrollo, ya que el objetivo principal sigue siendo una producción dirigida a un mercado, sólo que ahora se pretende que el capital ecológico de los lugares, antes olvidado, se tome en cuenta y lo aprovechen los propios pobladores del lugar (Viola, 2000: 26; Leff, 2002: 67; Toledo, 2003: 37). 


\section{Conclusiones}

Analizar el riesgo ambiental es un tema de gran complejidad para la sociedad actual porque trasciende el plano ecológico y afecta la vida cotidiana en los ámbitos sociocultural y económico. Este riesgo en las comunidades rurales merece especial interés ya que el manejo del ambiente constituye la principal estrategia para sobrevivir. En el Poblado 1 el deterioro ambiental causado por las acciones antropogénicas es el que provoca directamente la situación de riesgo, es decir, la destrucción ecológica y el agotamiento de los recursos naturales no son problemas generados netamente por factores naturales, sino que son creados y determinados por las formas sociales y patrones tecnológicos de apropiación y explotación económica de la naturaleza, que para el caso de las comunidades étnicas son formas no propias de su cultura, que tratan de incorporarlos a la dinámica nacional del progreso (Leff, 2002: 49; Toledo, 2003: 27).

Aunque este artículo se enfoca a las actividades y concepciones propias de los habitantes del Poblado 1, debemos insistir en el hecho de que en la localidad hay desigualdades causadas en gran parte por grandes productores que viven en otras localidades. Dichas actividades se han desarrollado históricamente y constituyen un factor de deterioro que incapacita a los pobladores para actuar de manera significativa para contrarrestar el riesgo ambiental. La situación de riesgo en el Poblado 1 aparece como una respuesta para satisfacer necesidades de mercado sin considerar las alteraciones al medio ambiente. En términos generales, los miembros del Poblado 1 intentan sobrevivir a las situaciones de riesgo ampliando su base agropecuaria-productiva, al mismo tiempo que disminuyen los pocos manchones de vegetación actual. La otra estrategia que llevan a cabo es la migración hacia las ciudades, con lo que se puede prever la pérdida de sustentabilidad alimenticia, el aumento de una población vieja y la desaparición del conocimiento local debido a que no hay receptores del mismo. Por desgracia, la situación descrita para el Poblado 1 no es única, pues creemos que la situación de riesgo en relación con el ambiente descrita en este trabajo se puede trasladar a otros poblados en la sierra totonaca.

Esto de ninguna manera significa que no hay ejemplos o acciones que se estén llevando a cabo en esta zona para contrarrestar las dificultades en que viven los totonacos. Sin embargo, es muy claro que el reto de la disminución del riesgo ambiental en 
poblaciones rurales es enorme y nos incluye a todos los habitantes, incluso a los de las grandes urbes. Las poblaciones rurales se deben atender, escuchar sus necesidades locales y tomar en cuenta los contextos culturales en que viven. Los modos de producción agrícola actual se deben reformular y hacer nuevas propuestas para mejorar las condiciones actuales de los habitantes, sin descuidar el uso del suelo y su fertilidad. Además, la migración es un fenómeno que cada vez nos afecta más. Veracruz está incrementando su tasa de migración que, de acuerdo con el último censo, indica que aproximadamente 800 mil personas salieron del estado en cinco años, lo que representan $10 \%$ de su población total (INEGI, 2000). En particular la migración juvenil trastoca de manera muy importante la dinámica social de los pueblos. Una vez que no hay jóvenes, es muy difícil dar continuidad social a la comunidad, los modos de organización para resolver problemas y la realización de obras al interior se paralizan o desarticulan. También es claro que para disminuir esta problemática las soluciones deben comprender políticas públicas nacionales y regionales, pero también soluciones locales. Aún más, sin una participación colectiva y articulada no se pueden poner en práctica acciones a favor de un verdadero desarrollo sustentable y una disminución real del riesgo, ya que finalmente somos nosotros quienes determinamos las propias condiciones de existencia.

\section{Agradecimientos}

Queremos agradecer a los miembros del Poblado 1 y a los traductores por su participación, tiempo y opiniones en esta investigación. También reconocer la ayuda del Programa de Mejoramiento de Profesorado (Promep), de la Secretaría de Educación Pública, por el financiamiento que nos otorgaron a través del rubro de Apoyo y Generación del Conocimiento, al proyecto "Evaluación del riesgo ambiental entre la población totonaca".

\section{Bibliografía}

Beck, Ulrich (1991), "Politics in risk society", en Ulrich Beck (ed.), Ecological enlightenment, Prometheus, Nueva Jersey, Estados Unidos, pp. 1-18.

Beck, Ulrich (1999), "Teoría de la sociedad del riesgo", en A. Giddens, Zygmunt Bauman, Niklas Luhmann y Beck Ulri- 
ch (eds.), Las consecuencias perversas de la modernidad, Antrhopos, Madrid, España, pp. 201-222.

Beck-Gernsheim, Elisabeth (1996), "Life as a Planning Project", en Scott Lash, Bronislaw Szerszynski y Brian Wynne (eds.), Risk, Environment and Modernity: Towards a New Ecology, Sage, Londres, Inglaterra, pp. 139-153.

Bell, Simon y Stephen Morse (1999), Sustainability indicators, measuring the immeasurable?, Earthcan, Londres, Inglaterra.

Dávila Garibi, Gabriela (coord.) (2000), Estado del desarrollo económico y social de los pueblos indígenas de México, tomos 1 y 2, primer informe, PNUD-INI, México.

Douglas, Marie y Aaron Wildavsky (1982), Risk and culture. An essay on the selection of technological and environmental dangers, University of California Press, California.

Dubos, René (1985), Celebraciones de la vida, Conacyt-Fondo de Cultura Económica, México.

Foucault, Michel (2004), Las palabras y las cosas. Una arqueología de las ciencias humanas, Siglo XXI, México.

Gomezjara, Francisco (dir. y coord.) (1998), "Zozocolco de Hidalgo", Enciclopedia municipal veracruzana, Gobierno del Estado de Veracruz, Jalapa, México, pp. 1-20.

Ichón, Alan (1973), La religión de los totonacas de la sierra, Instituto Nacional Indigenista, México.

INEGI (Instituto Nacional de Estadística, Geografía e Informática) (1998), Estadísticas del medio ambiente. México, 1997, INEGI-Semarnap, México.

INEGI (Instituto Nacional de Estadística, Geografía e Informática) (2000), Censo de Población, INEGI, México.

Leff, Enrique (2002), Saber ambiental. Sustentabilidad, racionalidad, complejidad, poder, Siglo XXI-pnuma-Centro de 
Investigaciones Interdiciplinarias en Ciencias y Humanidades, México.

Mires, Fernando (1996), La revolución que nadie soñó, o la otra posmodernidad, Nueva Sociedad, Caracas, Venezuela.

O'Connor, James (2001), Causas naturales. Ensayos de marxismo ecológico, Siglo XXI, México.

Ortiz Espejel, Benjamín (1990), “Los paisajes agrícolas del Totonacapan”, en Teresa Rojas Rabiela (coord.), Agricultura indigena: pasado y presente, Ediciones de la Casa Chata, México, pp. 359-397.

Pemex (Petróleos Mexicanos) (1999), Aspectos y datos fundamentales del Totonacapan, Informe Pemex, México.

Ramírez Melgarejo, Ramón (2002), La política del Estado mexicano en los procesos agrícolas y agrarios de los totonacos, Universidad Veracruzana, Veracruz, México.

Roma, Pepa (2002), Jaque a la globalización, Grijalbo, Madrid.

Segura Sebastián, Gaudencio (2002), Un pueblo llamado "San Miguel Tonatico”, Xalapa, Municipio de Zozocolco, Veracruz.

Toledo, Víctor (2003), Ecología, espiritualidad y conocimiento. De la sociedad del riesgo a la sociedad sustentable, Universidad Iberoamericana-PNumA, México.

Vaugh Switzer, Jaqueline (2001), Enviromental politics. Domestic and global dimensions, Bedford Martins, Washington, D. C.

Vergara Tenorio, María del Carmen (2000), "Agricultural environmental risk and rural community culture", tesis doctoral, University of Illinois at Urbana-Champaign, 145p.

Villarreal González, Diana (2000), La política económica y social de México en la globalización, UAM-Porrúa, México. 
Viola, Andreu (comp.) (2000), Antropología del desarrollo. Teorías y estudios etnográficos en América Latina, Paidós, Madrid.

Recibido: 19 de septiembre de 2006. Reenviado: 26 de octubre de 2007. Aceptado: 22 de febrero de 2008.

María del Carmen Vergara Tenorio. Es doctora en desarrollo humano y comunidades por la Universidad de Illinois, Urbana-Champaign IL-USA. Tiene especialidad en comunidad y estudios rurales, así como en roles de género en desarrollo internacional. Investigadora del Centro de Investigaciones Tropicales de la Universidad Veracruzana. Su línea de investigación actual se centra en: desarrollo rural, planeación y manejo de recursos naturales. Entre sus publicaciones recientes destacan: en coautoría, "Analysis of a natural resources management system in the Calakmul Biosphere Reserve", Landscape and Urban Planning, 74, pp. 223241 (2006); en coautoría, "Desarrollo económico regional y la construcción de empresas rurales comunitarias", en Pablo Alberto Torres Lima (coord.), Desarrollo regional y sustentabilidad en México”, El Colegio de Sonora-Universidad Autónoma MetropolitanaConacyt, Hermosillo, Son., pp. 383-404 (2005); en coautoría, "Diagnostic, Use Consciousness and Availability of Timber in three Micro Regions of Southeast. Mexico”, Bridging Scales \& Epistemologies: Linking Local Knowledge with Global Science in Multiple Scale Assessments. Conference Proceedings, 18 de marzo de 2004; en coautoría, "The Orchard System: An Intermediary Stage between Intensive Production and Natural Protection Areas", Sociedades Rurales, Producción y Medio Ambiente, 3(1), pp. 65-74 (2002).

Juan Roberto Cervantes Vázquez. Es licenciado en antropología social por la Universidad Veracruzana, Escuela Gestalt de Diseño. Su línea de investigación es: consumo cultural y psicología social. 